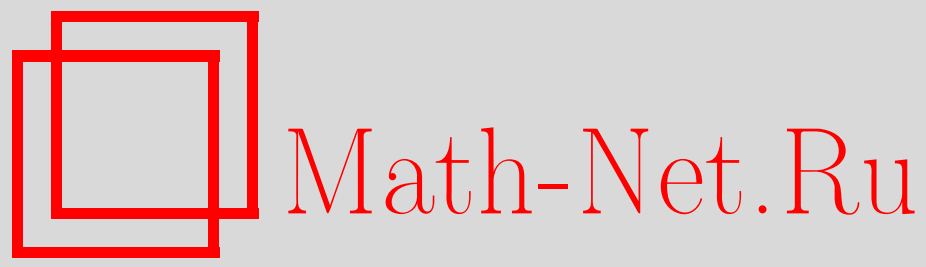

C. B. Пастухов, О некоторых вероятностно-статистических методах в техническом анализе, Теория вероятн. и ее примен., 2004, том 49, выпуск 2, 297-316

DOI: https://doi.org/10.4213/tvp220

Использование Общероссийского математического портала Math-Net.Ru подразумевает, что вы прочитали и согласны с пользовательским соглашением

http: //www . mathnet.ru/rus/agreement

Параметры загрузки:

IP : 54.162 .127 .20

26 апреля 2023 г., $15: 28: 58$

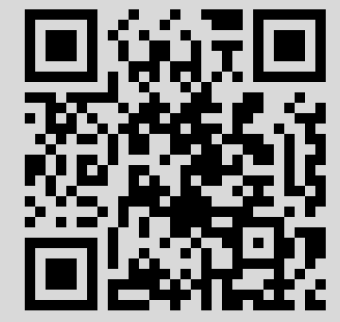




\section{О НЕКОТОРЫХ ВЕРОЯТНОСТНО-СТАТИСТИЧЕСКИХ МЕТОДАХ В ТЕХНИЧЕСКОМ АНАЛИЗЕ}

Дается математическое обоснование некоторых методов технического анализа, используемых на финансовом рынке. В частности, при помоши вводимого понятия $H$-волатильности $(H>0)$ обосновываются и корректируются так называемые renko-, kagi-модели. В случае винеровского процесса $H$-волатильность обладает рядом свойств, которые имеют место и на финансовом рынке при некоторых отличиях, указывающих на существование арбитражных возможностей. Извлечь данный арбитраж удается посредством вводимых renko-, kagi- $H$-стратегий.

Ключевые слова и фразы: $\Delta$-вариация, $\Delta$-волатильность, $H$-вариация, $H$-флуктуация, renko- $H$-инверсия, kagi- $H$-инверсия, renko- $H$-волатильность, kagi- $H$-волатильность, renko- $H$-стратегия, kagi- $H$-стратегия.

1. Введение. Для процесса $X=(X(t))_{t \geqslant 0}$, в отношении которого предполагается лишь непрерывность траекторий, в настоящей работе вводится понятие $H$-волатильности для $H>0$ (см. также [3]). В случае, когда процесс $X$ описывает поведение цены некоторого финансового актива, данное понятие позволяет строить стратегии, направленные на извлечение имеющихся на рынке арбитражных возможностей.

В основе $H$-волатильности лежат вариационные характеристики (1) и (2) (см. ниже), которые задают два подхода к данному понятию renko, kagi соответственно (происхождение этих понятий, связанных с построениями, применявшимися на японском рынке еще в XIX веке, пояснено в п. 2). Вычисление величин (1), (2) напрямую связано с процессами $|X(t)-X(0)|, \max _{[0, t]} X-X(t)$ соответственно, что позволяет представить их в удобном для вычисления виде (см. лемму 1 ), а также провести соответствующую нормировку. Следует отметить, что в финансовой математике давно используется понятие $\Delta$-волатильности (см. [5, с. 419]), в основе которого лежат вариационные характеристики (см. (3)), подобные указанным выше. Учитывая некоторую схожесть в

* Московский государственный университет им. М.В. Ломоносова, Ленинские горы, 119992 Москва, Россия; e-mail: acl@misis.ru 
определении данных понятий, представляется естественным использование термина «H-волатильность» наряду с уже существующим термином « $\Delta$-волатильность». Отметим также, что в отличие от «римановского» подхода при определении $\Delta$-волатильности, когда значения $X$ группируются по временно́й оси с интервалом $\Delta$, при определении $H$-волатильности используется «лебеговский» подход. Иначе говоря, существенным является лишь изменение $X$ на величину, не меньшую $H$, а время, за которое это изменение произошло, не имеет значения.

Если в качестве $X$ взять винеровский процесс, то для $H$-волатильности выполнены некоторые предельные свойства (см. теорему в п. 3). Оказывается, что подобные соотношения имеют место и на финансовом рынке, но пределы для многих активов отличаются от $2 H$ (предельного значения $H$-волатильности винеровского процесса). Это обстоятельство наводит на мысль, что если винеровский процесс соответствует безарбитражному случаю, то отличие $H$-волатильности от $2 H$ может говорить о существовании арбитражных возможностей для данного актива. Действительно, в этом случае удается построить стратегии, позволяющие получить в среднем положительный доход при нулевом начальном капитале (см. утверждения 2 и 3 ). Суть данных стратегий состоит в следующем: если $H$-волатильность больше $2 H$, то инвестор действует сонаправленно с рынком, т.е. в определенные моменты времени покупает, когда цена актива $X$ растет, и продает, когда цена падает. Если же $H$-волатильность меньше $2 H$, то инвестор действует против рынка, т.е. покупает при падении цены актива $X$ и продает в моменты роста.

Как будет показано ниже, для процесса $X$, описывающего поведение цены финансового актива, $H$-волатильность является своего рода фрактальной характеристикой и поэтому мало соответствует нашему интуитивному представлению о волатильности как мере изменчивости рынка. Иначе говоря, для «бурных» и «спокойных» дней $H$-волатильность принимает достаточно близкие друг к другу значения. В связи с этим в настоящей работе вводится понятие $H$-инверсии (см. также [3]), которое может быть использовано для определения волатильности (изменчивости) процесса $X$.

Статья имеет следующую структуру. В п. 2 вводится определение $H$-волатильности и $H$-инверсии, в п. 3 приведены результаты для винеровского процесса, п. 4 содержит описание стратегий, основанных на $H$-волатильности, в п. 5 при помощи $H$-инверсии определяется понятие волатильности (изменчивости), п. 6 содержит статистический анализ, а в п. 7 приведены доказательства основных результатов.

2. Определение $H$-волатильности и $H$-инверсия. Пусть процесс $X=(X(t))_{t \geqslant 0}$ задан на некотором стохастическом базисе 
$\left(\Omega, \mathscr{F},\left(\mathscr{F}_{t}\right)_{t \geqslant 0}, \mathbf{P}\right)$ и принимает значения в $\mathbf{R}^{1}$. Здесь и далее полагаем, что траектории $X$ являются непрерывными функциями. Понятие $H$-волатильности основано на следующих вариационных характеристиках процесса $X$. Для произвольных $H, T>0$ рассмотрим величины

$$
\begin{aligned}
& U_{T}(H, X)=\sup _{\mathbf{T}_{1}} \sum_{k=1}^{K}\left|X\left(t_{k}\right)-X\left(t_{k-1}\right)\right|, \\
& V_{T}(H, X)=\sup _{\mathbf{T}_{2}} \sum_{l=1}^{L}\left|X\left(t_{l}\right)-X\left(t_{l-1}\right)\right|,
\end{aligned}
$$

где $\mathbf{T}_{1}-$ множество всех конечных разбиений $\left(t_{0}, t_{1}, \ldots, t_{K}\right)$ таких, что $0=t_{0}<t_{1}<\cdots<t_{K} \leqslant T$ и $\left|X\left(t_{k}\right)-X\left(t_{k-1}\right)\right| / H \in \mathbf{N}=\{1,2, \ldots\}$ для $k=1, \ldots, K, \mathbf{T}_{2}-$ множество всех конечных разбиений $\left(t_{0}, t_{1}, \ldots, t_{L}\right)$ таких, что $0 \leqslant t_{0}<t_{1}<\cdots<t_{L} \leqslant T$ и $\left|X\left(t_{l}\right)-X\left(t_{l-1}\right)\right| / H \in[1,+\infty)$ для $l=1, \ldots, L$.

Величину (1) будем называть $H$-флуктуачией реализации процесса $X$ на $[0, T]$, а величину (2) - H-вариаиией реализации процесса $X$ на $[0, T]$. Если для какого-то $H>0$ не существует соответствующих разбиений из (1), (2), полагаем указанные величины равными нулю. Для сравнения обратимся к $\Delta$-вариации (см. [5, с. 419]), широко используемой в финансовой математике, которая есть по определению

$$
Z_{T}(\Delta, X)=\sum_{k=1}^{[T / \Delta]}|X(k \Delta)-X((k-1) \Delta)|
$$

где $\Delta, T>0$, а [ [ ] означает взятие целой части. Схожесть величин $(1),(2)$ и (3) состоит в том, что для «достаточно регулярных» реализаций процесса $X$ они при малых $H$ и $\Delta$ соответственно близки к полной вариации, которая есть по определению

$$
\sup \sum_{n=1}^{N}\left|X\left(t_{n}\right)-X\left(t_{n-1}\right)\right|
$$

где супремум берется по разбиениям $\left(t_{0}, t_{1}, \ldots, t_{N}\right)$ таким, что $0 \leqslant t_{0}<$ $t_{1}<\cdots<t_{N} \leqslant T$. Принципиальным же отличием, о котором говорилось выше, является то, что в случае $\Delta$-вариации используется так называемый «римановский» подход, в то время как в настоящей работе предлагается «лебеговский» вариант построения вариационных характеристик. Кроме того, при использовании величин (1), (2) на финансовом рынке процесс $X$ определяет непосредственно цены того или иного актива, а не их логарифмы, как в случае величины (3).

При статистическом анализе процесса $X$ обычно оперируют не с (3), а с нормированной величиной

$$
\zeta_{T}(\Delta, X)=\frac{Z_{T}(\Delta, X)}{[T / \Delta]}
$$


которую принято называть $\Delta$-волатильностью. По аналогии с $\zeta_{T}(\Delta, X)$ естественно было бы нормировать величины (1), (2), однако встает проблема о нормирующем коэффициенте. Ответ на это вопрос дает следующая лемма.

Лемма 1. Пусть реализачии прочесса $X$ являются непрерывными функииями на $[0, T]$. Тогда для любого $H>0$ существуют величины $M=M_{T}(H, X)$ и $N=N_{T}(H, X)$, а также последовательности $\left(\rho_{m}^{*}, \rho_{m}\right)_{m=0,1, \ldots, M} u\left(\tau_{n}^{*}, \tau_{n}\right)_{n=0,1, \ldots, N}$ maкue, uто $\left(\rho_{m}\right)_{m=0,1, \ldots, M}$, $\left(\tau_{n}\right)_{n=0,1, \ldots, N}-$ марковские, а величины $\rho_{m}^{*}, \tau_{n}^{*}$, определяемые, исходя из поведения прочесса $X$ на интервалах $\left[\rho_{m-1}, \rho_{m}\right],\left[\tau_{n-1}, \tau_{n}\right]$ соответственно $\left(\rho_{-1}=\tau_{-1}=0\right)$, удовлетворяют равенствам

$$
\begin{aligned}
& U_{T}(H, X)=\sum_{m=1}^{M}\left|X\left(\rho_{m}^{*}\right)-X\left(\rho_{m-1}^{*}\right)\right|, \\
& V_{T}(H, X)=\sum_{n=1}^{N}\left|X\left(\tau_{n}^{*}\right)-X\left(\tau_{n-1}^{*}\right)\right| .
\end{aligned}
$$

Построения моментов $\left(\rho_{m}^{*}, \rho_{m}\right),\left(\tau_{n}^{*}, \tau_{n}\right)$ из леммы 1 с целью прогноза дальнейшего поведения цены финансового актива широко используются в техническом анализе и называются соответственно renko- и kagi-методами. Впервые подобные построения стали использовать в Японии, по всей видимости, еще в XIX веке, когда рынок только зарождался (см. [7]). Поэтому термины renko (точнее было бы писать renga) и kagi имеют японские корни и означают соответственно кирпич и ключ (предел, ... ). Среди существующих на данный момент моделей, основанных на kagi- и renko-построениях, наиболее общей и строгой с математической точки зрения представляется модель, описанная в работе [2], где для построения прогноза использовалась теория кусочно-монотонных аппроксимаций и, в частности, результаты работы [4]. Здесь же заметим, что величины $M_{T}(H, X), N_{T}(H, X)$ из леммы 1 тесно связаны с указанным аппаратом кусочно-монотонных аппроксимаций (подробнее см. замечание к доказательству леммы 1 в п. 7).

Величину $M_{T}(H, X)$ будем называть renko- $H$-uнверсией, а величину $N_{T}(H, X)-k a g i-H$-инверсией реализации процесса $X$ на $[0, T]$. Учитывая вышесказанное, отметим, что использование терминов renko, kagi представляется целесообразным. Как будет показано ниже (см. п. 5), $H$-инверсия может служить хорошей характеристикой волатильности (меры изменчивости) рынка и использоваться для оценки различных рыночных показателей. Из леммы 1 становится понятно, что величины $M_{T}(H, X)$ и $N_{T}(H, X)$ вполне естественно брать в качестве нормирующих коэффициентов для (1) и (2) соответственно. По аналогии с (4) 
определим

$$
\begin{aligned}
\xi_{T}(H, X) & =\frac{U_{T}(H, X)}{M_{T}(H, X)}, \\
\eta_{T}(H, X) & =\frac{V_{T}(H, X)}{N_{T}(H, X)} .
\end{aligned}
$$

Величину (7) будем называть renko-H-волатильностью, а величину (8) - kagi-H-волатильностью реализации процесса $X$ на $[0, T]$. Наряду с величинами (7), (8) для произвольного $p \geqslant 1$ будем рассматривать также величины

$$
\begin{aligned}
\xi_{T}^{(p)}(H, X) & =\frac{U_{T}^{(p)}(H, X)}{M_{T}(H, X)} \\
\eta_{T}^{(p)}(H, X) & =\frac{V_{T}^{(p)}(H, X)}{N_{T}(H, X)}
\end{aligned}
$$

где $U_{T}^{(p)}(H, X), V_{T}^{(p)}(H, X)$ определяются из соотношений

$$
\begin{aligned}
& U_{T}^{(p)}(H, X)=\sum_{m=1}^{M}\left|X\left(\rho_{m}^{*}\right)-X\left(\rho_{m-1}^{*}\right)\right|^{p} \\
& V_{T}^{(p)}(H, X)=\sum_{n=1}^{N}\left|X\left(\tau_{n}^{*}\right)-X\left(\tau_{n-1}^{*}\right)\right|^{p}
\end{aligned}
$$

$M=M_{T}(H, X), N=N_{T}(H, X),\left(\rho_{m}^{*}\right)_{m=0,1, \ldots, M}$ и $\left(\tau_{n}^{*}\right)_{n=0,1, \ldots, N}$ - те же, что и в лемме 1 . Величину (9) будем называть renko-H-волатильностью порядка $p$, величину (10) - kagi-H-волатильностью порядка $p$ процесса $X$ на $[0, T]$.

\section{3. Некоторые предельные свойства винеровского процесса.} Для renko-, kagi- $H$-волатильности порядка $p$ винеровского процесса $W=\left(W_{t}\right)_{t \geqslant 0}$ выполнено следующее.

Теорема. Пусть $W-$ стандартньй винеровский прочесс, тогда для любых $p \geqslant 1, H>0, \sigma>0$ выполнено

$$
\begin{aligned}
& \lim _{T \rightarrow+\infty} \xi_{T}^{(p)}(H, \sigma W)=R_{W}(p) H^{p} \quad \text { (n.н.), } \\
& \lim _{T \rightarrow+\infty} \eta_{T}^{(p)}(H, \sigma W)=K_{W}(p) H^{p} \quad \text { (n.н.) }
\end{aligned}
$$

где $R_{W}(p)=\sum_{n=1}^{\infty} n^{p} / 2^{n}, K_{W}(p)=\mathbf{E}\left(2+W_{\theta} / H\right)^{p}, \theta=\inf \{u \geqslant 0$ : $\left.\max _{[0, u]} W-W_{u}=H\right\}$.

Случай $p=1$ для величины $\eta_{T}^{(p)}(H, W)$ был рассмотрен в работе [3]. Схожесть в пределе у обеих $H$-волатильностей может быть 
объяснена тем, что renko- и kagi-построения напрямую связаны с процессами $|X(t)-X(0)|$ и $\max _{[0, t]} X-X(t)$ соответственно (см. доказательство леммы 1$)$. В случае, когда $X$ есть броуновское движение, указанные процессы эквиваленты по распределению в силу теоремы Леви [6, с. 211]. В частности, для $p=1$ пределы совпадают, поскольку $R_{W}(1)=K_{W}(1)=2$ (см. замечание к доказательству теоремы в п. 7). Здесь же заметим, что величины $R_{W}(p), K_{W}(p)$ не зависят от $\sigma$, т.е. $H$-волатильность (в пределе) является инвариантом относительно растяжения и сжатия винеровского процесса. В дальнейшем будет показано, что подобным свойством обладает и финансовый рынок (см. п. 6). Для renko-, kagi- $H$ инверсии винеровского процесса выполнено следующее.

Лемма 2. Пусть $W-$ стандартный винеровский прочесс, тогда для любих $H, \sigma>0$ выполнено

$$
\begin{aligned}
& \lim _{T \rightarrow+\infty} \frac{T}{M_{T}(H, \sigma W)}=\frac{2 H^{2}}{\sigma^{2}} \quad \text { (n.н.), } \\
& \lim _{T \rightarrow+\infty} \frac{T}{N_{T}(H, \sigma W)}=\frac{H^{2}}{\sigma^{2}} \quad \text { (n.н.). }
\end{aligned}
$$

Отсюда видно, что, в отличие от $H$-волатильности, $H$-инверсия процесса $\sigma W$ напрямую зависит от параметра $\sigma$. И, как будет показано ниже (см. п. 5), данное обстоятельство дает основание для использования $H$-инверсии в качестве оценки волатильности (как меры изменчивости) рынка. Интересно также отметить следствие из теоремы и леммы 2.

Следствие 1. Пусть $W$ - стандартный винеровский прочесс, тогда для любых $p \geqslant 1, H>0, \sigma>0$ выполнено

$$
\begin{gathered}
\lim _{T \rightarrow+\infty} \frac{2 U_{T}^{(p)}(H, \sigma W)}{T}=\sigma^{2} R_{W}(p) H^{p-2} \quad \text { (п.н.) }, \\
\lim _{T \rightarrow+\infty} \frac{V_{T}^{(p)}(H, \sigma W)}{T}=\sigma^{2} K_{W}(p) H^{p-2} \quad \text { (n.н.). }
\end{gathered}
$$

4. Renko-, kagi-H-стратегии. Оказывается, что соотношения, подобные $(13),(14)$, имеют место и на финансовом рынке. При статистическом анализе процесса $X$, определяюшего цены некоторого актива, для случаев $p=1,2$, как наиболее важных с практической точки зрения, для достаточного большого $T$ выявлено следующее:

$$
\begin{aligned}
& \xi_{T}^{(p)}(H, X) \approx C_{R}(p) H^{p}, \\
& \eta_{T}^{(p)}(H, X) \approx C_{K}(p) H^{p},
\end{aligned}
$$

где величины $C_{R}(p)=C_{R}(p, X), C_{K}(p)=C_{K}(p, X)$ для заданных $X$ и $p$ можно в первом приближении считать константами в зависимости 
от $H$ (подробнее см. п. 6). В дальнейшем в данном пункте рассматривается только случай $p=1$, поэтому для простоты обозначений индекс $p=1$ в соответствующих величинах будем опускать. Для многих финансовых активов значения $C_{R}(1), C_{K}(1)$ отличаются от значений $R_{W}(1)=K_{W}(1)=2$ (константы для винеровского процесса, см. замечание к доказательству теоремы в п. 7). Это обстоятельство наводит на мысль, что если винеровский процесс соответствует безарбитражному случаю, то отличие $C_{R}(1), C_{K}(1)$ от 2 , возможно, указывает на наличие арбитража для данного $X$. Оказывается, что в этом случае знание $H$-волатильности (а точнее, ее предельного значения) позволяет построить стратегии с положительным в среднем доходом при нулевом начальном капитале.

В настоящей работе мы полагаем, что операционные издержки равны нулю и инвестор имеет возможность «коротко» продавать актив $X$ (т.е. брать его взаймы с целью продажи), а также что ставка за взятие взаймы денег и актива равна нулю (предположение о нулевой процентной ставке выглядит вполне адекватным, если речь идет, например, о внутридневной торговле). На интервале $[0, T]$ рассмотрим последовательности $\left(\rho_{m}^{*}, \rho_{m}\right)_{m=0, \ldots, M_{T}(H, X)},\left(\tau_{n}^{*}, \tau_{n}\right)_{n=0, \ldots, N_{T}(H, X)}$, о которых говорится в лемме 1 , и определим процессы $\widetilde{\gamma}^{R}=\left(\widetilde{\gamma}_{t}^{R}(H, X)\right)_{0 \leqslant t \leqslant T}$, $\widetilde{\gamma}^{K}=\left(\widetilde{\gamma}_{t}^{K}(H, X)\right)_{0 \leqslant t \leqslant T}$, которые будут задавать количество актива $X$ в портфеле инвестора, следующим образом:

$$
\begin{aligned}
& \tilde{\gamma}_{t}^{R}(H, X)=\sum_{m=1}^{M_{T}(H, X)} \operatorname{sign}\left(X\left(\rho_{m-1}\right)-X\left(\rho_{m-1}^{*}\right)\right) \chi_{\left[\rho_{m-1}, \rho_{m}\right)}(t), \\
& \tilde{\gamma}_{t}^{K}(H, X)=\sum_{n=1}^{N_{T}(H, X)} \operatorname{sign}\left(X\left(\tau_{n-1}\right)-X\left(\tau_{n-1}^{*}\right)\right) \chi_{\left[\tau_{n-1}, \tau_{n}\right)}(t),
\end{aligned}
$$

где $\chi_{A}(t)$ - характеристическая функция множества $A$. Процессы $\widetilde{\gamma}^{R}$ и $\widetilde{\gamma}^{K}$ будем называть соответственно renko ${ }^{+}-H$-cmpamezuеŭ и $k_{\text {agi }}{ }^{+}$$H$-стратегией для процесса $X$ на $[0, T]$. Иными словами, renko ${ }^{+} H$ стратегия состоит в том, что в момент $\rho_{m}$ покупаем единицу актива $X$, если $X\left(\rho_{m}\right)-X\left(\rho_{m}^{*}\right)>0$, и продаем, если $X\left(\rho_{m}\right)-X\left(\rho_{m}^{*}\right)<0$. То есть в каждый момент времени на интервале $\left[\rho_{0}, \rho_{M}\right)$ в портфеле инвестора содержится единица актива $X$ со знаком, а переформирование происходит в марковские моменты $\left(\rho_{m}\right)_{m=0, \ldots, M}$. Указанная стратегия широко используется на практике (см. [7]), однако, как будет показано ниже (см. утверждение 1), для некоторого рода финансовых активов ее применение заведомо приведет к убыткам. Смысл $\mathrm{kagi}^{+}-H$-стратегии состоит в том же. Капитал в момент времени $t \in[0, T]$, соответствующий $\tilde{\gamma}^{R}, \tilde{\gamma}^{K}$, будем обозначать через

$$
\tilde{Y}_{t}^{R}(H, X)=\int_{0}^{t} \widetilde{\gamma}_{u}^{R}(H, X) d X(u), \quad \tilde{Y}_{t}^{K}(H, X)=\int_{0}^{t} \widetilde{\gamma}_{u}^{K}(H, X) d X(u) .
$$


Значение капитала в момент времени $T$ есть доход от указанных стратегий на $[0, T]$.

Утверждение 1. Доход от renko ${ }^{+}, k_{\text {agi }}{ }^{+}-H$-cmpameаuй (21), (22) для прочесса $X$ на интервале $[0, T]$ равен соответственно

$$
\begin{aligned}
& \tilde{Y}_{T}^{R}(H, X)=\left(\xi_{T}(H, X)-2 H\right) M_{T}(H, X)+\varepsilon_{T}^{R}(H, X), \\
& \tilde{Y}_{T}^{K}(H, X)=\left(\eta_{T}(H, X)-2 H\right) N_{T}(H, X)+\varepsilon_{T}^{K}(H, X),
\end{aligned}
$$

где $0<\varepsilon_{T}^{R}(H, X)<2 H, 0<\varepsilon_{T}^{K}(H, X) \leqslant H$.

Отсюда сразу следует, что если эмпирическая $H$-волатильность для некоторого актива меньше $2 H$ (например, для фьючерсов на индексы SP500, Nasdaq100, см. п. 6), то применение $\tilde{\gamma}^{R}, \widetilde{\gamma}^{K}$ убыточно. В этом случае, как несложно заметить, целесообразней использовать обратные стратегии, т.е. стратегии $\left(-\widetilde{\gamma}^{R}\right),\left(-\widetilde{\gamma}^{K}\right)$. Следующие утверждения содержат точные формулировки на этот счет. Скажем, что процесс $X$ обладает свойством renko, если выполнено

$$
\lim _{T \rightarrow+\infty} \mathbf{E} \xi_{T}(H, X)=R(H) H .
$$

Скажем, что процесс $X$ обладает свойством kagi, если выполнено

$$
\lim _{T \rightarrow+\infty} \mathbf{E} \eta_{T}(H, X)=K(H) H .
$$

Заметим, что с учетом соотношений (19), (20) данные свойства выглядят вполне естественными. Определим процессы $\gamma^{R}=\left(\gamma_{t}^{R}(H, X)\right)_{0 \leqslant t \leqslant T}$, $\gamma^{K}=\left(\gamma_{t}^{K}(H, X)\right)_{0 \leqslant t \leqslant T}$ следующим образом:

$$
\begin{aligned}
\gamma_{t}^{R}(H, X) & =\widetilde{\gamma}_{t}^{R}(H, X)\left(\chi_{\{R(H) \geqslant 2\}}-\chi_{\{R(H)<2\}}\right), \\
\gamma_{t}^{K}(H, X) & =\widetilde{\gamma}_{t}^{K}(H, X)\left(\chi_{\{K(H) \geqslant 2\}}-\chi_{\{K(H)<2\}}\right),
\end{aligned}
$$

где $\tilde{\gamma}^{R}, \widetilde{\gamma}^{K}$ имеют вид (21) и (22) соответственно. Тогда процессы $\gamma^{R}$ и $\gamma^{K}$ будем называть соответственно renko-H-cmpameәuеŭ и kagi-H-cmpaтегuей для процесса $X$ на $[0, T]$. Стратегию (25) можно интерпретировать следующим образом: если $R(H) \geqslant 2$, то инвестор должен действовать сонаправленно с рынком, т.е. в момент времени $\rho_{m}$ покупать, когда цена актива $X$ растет, и продавать, когда цена падает. Если же $R(H)<2$, то инвестор должен действовать против рынка, т.е. в момент времени $\rho_{m}$ покупать при падении цены и продавать в случае роста. Для kagi- $H$-стратегии все аналогично. Капитал в момент времени $t \in[0, T]$, соответствующий данным стратегиям, будем обозначать через

$$
Y_{t}^{R}(H, X)=\int_{0}^{t} \gamma_{u}^{R}(H, X) d X(u), \quad Y_{t}^{K}(H, X)=\int_{0}^{t} \gamma_{u}^{K}(H, X) d X(u) .
$$


Рассмотрим также величины

$$
y_{T}^{R}(H, X)=\frac{Y_{T}^{R}(H, X)}{M_{T}(H, X)}, \quad y_{T}^{K}(H, X)=\frac{Y_{T}^{K}(H, X)}{N_{T}(H, X)},
$$

которые будут определять средний доход одной транзакции на $[0, T]$ для стратегий $\gamma^{R}, \gamma^{K}$ соответственно.

Утверждение 2. Пусть прочесс $X$ oбладает renko-cвойcmвом (23), тогда для rепко-H-стратегии (25) выполнено

$$
\lim _{T \rightarrow+\infty} \mathbf{E} y_{T}^{R}(H, X)=|R(H)-2| H
$$

Заметим, что в случае $R(H)=2$ (в частности, для винеровского процесса) renko- $H$-стратегия не приносит прибыли, что вполне соответствует классическому пониманию безарбитражности рынка. Аналогичное утверждение выполняется и для kagi-H-стратегии.

Утверждение 3. Пусть прочесс $X$ обладает kagi-свойством (24), тогда для kagi-H-стратегии (26) выполнено

$$
\lim _{T \rightarrow+\infty} \mathbf{E} y_{T}^{K}(H, X)=|K(H)-2| H
$$

Напоследок отметим, что в силу (19), (20) величины $R(H), K(H)$ можно считать константами. А это в свою очередь позволяет говорить, что renko-, kagi- $H$-стратегии не зависят от $H$, а являются характеристическими свойствами рассматриваемого финансового инструмента.

5. $H$-инверсия как мера изменчивости (волатильность). Понятие $H$-волатильности, как показывает статистический анализ (см. п. 6), является некоторой фрактальной характеристикой финансового актива и слабо соответствует нашему интуитивному представлению о волатильности как мере изменчивости рынка. Иначе говоря, для «бурных» и «спокойных» дней значения $H$-волатильности мало отличаются друг от друга. В этом смысле термин «H-волатильность» не совсем адекватно отражает само понятие волатильности (тем не менее данная терминология представляется целесообразной ввиду аналогии с уже устоявшимся понятием $\Delta$-волатильности, о чем было сказано выше). В то же время, как будет показано ниже, хорошей характеристикой изменчивости рынка может служить $H$-инверсия. Из леммы 2 можно получить оценку параметра $\sigma$ (т.е. волатильности) для процесса $X=\sigma W$, где $W$ - стандартный винеровский процесс, по одному из выражений

$$
\sigma_{\text {renko }}=H \sqrt{\frac{2 M_{T}(H, X)}{T}}, \quad \sigma_{\text {kagi }}=H \sqrt{\frac{N_{T}(H, X)}{T}} .
$$


Для винеровского процесса данные оценки сходятся к одному и тому же значению при $T \rightarrow \infty$ для различных $H$. Оказывается, если $X$ описывает поведение цены финансового актива, то указанные выражения также можно считать в первом приближении константами в зависимости от $H$ для достаточно большого $T$ (см. п. 6). Это обстоятельство служит основанием для того, чтобы брать величины (27) в качестве оценки меры изменчивости произвольного финансового актива. Определяя таким образом волатильность, мы придаем данному понятию практическую наглядность. Например, знание kagi- $H$-инверсии (или $\sigma_{\text {kagi }}$ ) позволяет ответить на вопрос: сколько раз на интервале $[0, T]$ меняется направление изменения цен $X$, если пренебрегать изменениями на величину, меньшую $H$ ? При этом интуитивно понятно, что чем сильнее «лихорадит» рынок, тем больше значение kagi- $H$-инверсии, и наоборот. Renko$H$-инверсия дает похожую информацию, правда, иногда нагляднее с практической точки зрения брать не $M_{T}(H, X)$, а величину $\widetilde{M}_{T}(H, X)$. Определение $\widetilde{M}_{T}(H, X)$ дано в доказательстве леммы 1 (см. п. 7), здесь же только заметим, что величина $\widetilde{M}_{T}(H, X)$ говорит о том, сколько скачков размера $H$ у цены $X$ происходит на интервале $[0, T]$, если под скачком пониматє попадание цены на новый по отношению к предыдушему уровень вида $X(0)+k H$, где $k$ - целое число. Отметим также, что для винеровского процесса $W$ выполнено (см. доказательство леммы 2)

$$
\lim _{T \rightarrow+\infty} \frac{\widetilde{M}_{T}(H, W)}{M_{T}(H, W)}=2 \quad \text { (п.н.). }
$$

Иными словами, для оценки волатильности в (27) можно брать величину $\widetilde{M}_{T}(H, X)$ вместо $2 M_{T}(H, X)$. Статистический анализ финансового рынка показывает, что в обоих случаях величина $\sigma_{\text {renko }}$ принимает близкие значения, поэтому в настоящей работе мы ограничиваемся рассмотрением только $M_{T}(H, X)$.

Интересно также отметить в качестве одного из примеров использования $H$-инверсии на финансовом рынке следующее. Для произвольного процесса $X=(X(t))_{0 \leqslant t \leqslant T}$ обозначим

$$
R_{T}(X)=\max _{[0, T]} X-\min _{[0, T]} X
$$

Если в качестве $X$ взять процесс $\sigma W$, где $W$ - стандартное броуновское движение, а $\sigma>0$, то выполнено равенство (см., например, [1])

$$
\mathrm{E}\left(\frac{R_{T}(X)}{\sigma \sqrt{T}}\right)=\sqrt{\frac{8}{\pi}} \approx 1.596 .
$$

Для произвольного процесса $X$ в качестве $\sigma$ будем брать одно из выражений $(27)$, например $\sigma_{\text {kagi }}$. Обозначим тогда

$$
\omega_{T}^{\mathrm{kagi}}(H, X)=\frac{R_{T}(X)}{H \sqrt{N_{T}(H, X)}} .
$$


Оказывается, если $X$ описывает поведение цены некоторого финансового актива, то имеет место свойство (см. п. 6)

$$
\omega_{T}^{\mathrm{kagi}}(H, X) \approx C
$$

где константа $C=C(X)$ не зависит от $H$ и для многих активов близка к значению $\sqrt{\frac{8}{\pi}}$ (ср. с (28)). Отсюда, в частности, следует соотношение

$$
R_{T}(X) \approx C H \sqrt{N_{T}(H, X)}
$$

Подобным свойством обладает и renko- $H$-инверсия. Резюмируя вышесказанное, отметим, что рассматриваемое в настояшей статье понятие $H$-инверсии является удачной характеристикой финансового актива и может быть использовано для оценки разнообразных рыночных показателей. Также заметим, что оценку параметра $\sigma$ для процесса $X=\sigma W$, где $W$ - стандартное броуновское движение, можно получить также из следствия $1(p=2)$ по одному из выражений $\left(R_{W}(2)=6, K_{W}(2)=5\right.$, см. замечание к доказательству теоремы в п. 7)

$$
\sigma_{\text {renko }}^{(2)}=\sqrt{\frac{U_{T}^{(2)}(H, X)}{3 T}}, \quad \sigma_{\text {kagi }}^{(2)}=\sqrt{\frac{V_{T}^{(2)}(H, X)}{5 T}} .
$$

Статистический анализ финансового рынка показывает (см. п. 6), что если $X$ описывает поведение цен финансового актива, то данные выражения, так же как и выражения (27), можно считать константами в зависимости от $H$. При этом следует отметить, что величины (31) более устойчивы к изменениям $H$, чем (27), т.е. обладают меньшим квадратическим отклонением от среднего для различных $H$. Но, несмотря на это, $\sigma_{\text {renko }}, \sigma_{\text {kagi }}$ представляются более наглядными с практической точки зрения, к тому же в выражениях (31) присутствуют константы 3 и 5, которые возникли из предельных теорем для винеровского процесса, и, вообще говоря, другие для произвольного финансового актива.

6. Статистический анализ. Статистический анализ будем проводить для внутридневных цен фьючерса на индекс SP500 (EminiSP500 futures) и фьючерса на индекс Nasdaq-100 (Emini-Nasdaq100 futures), торгуемых на бирже CME (Chicago Mercantile Exchange), за 2002-2003 гг. (данный период содержит 471 торговых дней). При этом для каждого торгового дня выбираем наиболее ликвидный фьючерсный контракт, т.е. либо фьючерс с ближайшей к рассматриваемому дню датой погашения, либо следуюший за ним. Будем предполагать, что время $t$ изменяется дискретно с интервалом в 1 секунду, а в качестве $X(t)$ для определенности берем цену последней сделки в момент $t$. 
Для каждого $i$-го дня из указанного периода находим renko-, kagi- $H$ инверсию $M(i), N(i)$ и величины $U^{(1)}(i), U^{(2)}(i), V^{(1)}(i), V^{(2)}(i)$ по формулам (11), (12) соответственно, а также $\omega_{\text {kagi }}(i)$ из выражения (29). Для окончательных расчетов берем значения $M=\sum M(i), N=\sum N(i)$, $U^{(1)}=\sum U^{(1)}(i), U^{(2)}=\sum U^{(2)}(i), V^{(1)}=\sum V^{(1)}(i), V^{(2)}=\sum V^{(2)}(i)$, $\omega_{\text {kagi }}=\sum \omega_{\text {kagi }}(i) / \sum 1$, где суммирование ведется по всем $i$-м торговым дням. Значение $H$ измеряется в пунктах соответствующего фьючерса (изменение на 1 пункт цены фьючерса соответствует изменению денежной позиции на $\$ 50$ для Emini-SP500 и на $\$ 20$ для фьючерса EminiNasdaq100). В таблицах 1 и 2 приведены соответствуюшие характеристики для указанных фьючерсов для различных $H$, откуда, в частности, следуют соотношения (19), (20) и (30).

\begin{tabular}{|c|c|c|c|c|c|c|c|c|c|c|c|c|c|}
\hline$H$ & 1 & 1.25 & 1.5 & 1.75 & 2 & 2.25 & 2.5 & 2.75 & 3 & 3.25 & 3.5 & 3.75 & 4 \\
\hline$\xi^{(1)} / H$ & 1.83 & 1.84 & 1.86 & 1.88 & 1.88 & 1.86 & 1.88 & 1.84 & 1.83 & 1.80 & 1.79 & 1.75 & 1.69 \\
\hline$\xi^{(2)} / H^{2}$ & 4.86 & 4.91 & 5.04 & 5.17 & 5.21 & 5.06 & 5.14 & 4.86 & 4.83 & 4.57 & 4.54 & 4.26 & 3.94 \\
\hline$\sigma_{\text {renko }}$ & 22.9 & 22.7 & 22.5 & 22.3 & 22.1 & 22.6 & 22.7 & 23.4 & 23.5 & 24.7 & 25.0 & 26.0 & 27.4 \\
\hline$\sigma_{\text {renko }}^{(2)}$ & 20.6 & 20.6 & 20.6 & 20.7 & 20.6 & 20.8 & 21.0 & 21.0 & 21.1 & 21.6 & 21.8 & 21.9 & 22.2 \\
\hline$\eta^{(1)} / H$ & 1.83 & 1.85 & 1.85 & 1.87 & 1.89 & 1.91 & 1.93 & 1.93 & 1.92 & 1.90 & 1.89 & 1.87 & 1.87 \\
\hline$\eta^{(2)} / H^{2}$ & 4.23 & 4.28 & 4.31 & 4.38 & 4.47 & 4.54 & 4.63 & 4.61 & 4.55 & 4.46 & 4.38 & 4.32 & 4.30 \\
\hline$\sigma_{\text {kagi }}$ & 21.1 & 21.2 & 21.1 & 21.1 & 20.9 & 20.8 & 20.7 & 20.9 & 21.2 & 21.5 & 21.8 & 22.2 & 22.4 \\
\hline$\sigma_{\text {kagi }}^{(2)}$ & 19.5 & 19.6 & 19.6 & 19.7 & 19.8 & 19.9 & 20.0 & 20.0 & 20.2 & 20.3 & 20.4 & 20.6 & 20.8 \\
\hline$\omega_{\text {kagi }}$ & 1.49 & 1.51 & 1.52 & 1.54 & 1.57 & 1.59 & 1.61 & 1.60 & 1.58 & 1.58 & 1.55 & 1.58 & 1.60 \\
\hline
\end{tabular}

Таблица 1. Emini-SP500 futures

\begin{tabular}{|c|c|c|c|c|c|c|c|c|c|c|c|c|c|}
\hline$H$ & 2 & 2.5 & 3 & 3.5 & 4 & 4.5 & 5 & 5.5 & 6 & 6.5 & 7 & 7.5 & 8 \\
\hline$\xi^{(1)} / H$ & 1.85 & 1.86 & 1.86 & 1.87 & 1.89 & 1.87 & 1.86 & 1.83 & 1.83 & 1.79 & 1.76 & 1.72 & 1.70 \\
\hline$\xi^{(2)} / H^{2}$ & 4.95 & 5.02 & 5.00 & 5.07 & 5.13 & 5.06 & 4.95 & 4.77 & 4.73 & 4.57 & 4.36 & 4.14 & 4.06 \\
\hline$\sigma_{\text {renko }}$ & 46.0 & 45.4 & 45.4 & 45.1 & 45.0 & 45.9 & 46.2 & 47.4 & 48.7 & 50.6 & 51.7 & 54.2 & 54.4 \\
\hline$\sigma_{\text {renko }}^{(2)}$ & 41.8 & 41.5 & 41.5 & 41.4 & 41.6 & 42.1 & 42.0 & 42.2 & 43.3 & 44.2 & 44.1 & 45.1 & 44.7 \\
\hline$\eta^{(1)} / H$ & 1.86 & 1.88 & 1.89 & 1.89 & 1.89 & 1.89 & 1.90 & 1.91 & 1.92 & 1.91 & 1.90 & 1.89 & 1.87 \\
\hline$\eta^{(2)} / H^{2}$ & 4.40 & 4.45 & 4.44 & 4.43 & 4.43 & 4.44 & 4.47 & 4.52 & 4.53 & 4.48 & 4.43 & 4.36 & 4.24 \\
\hline$\sigma_{\text {kagi }}$ & 41.8 & 41.8 & 42.1 & 42.3 & 42.4 & 42.6 & 42.6 & 42.6 & 42.8 & 43.3 & 43.9 & 44.6 & 45.7 \\
\hline$\sigma_{\text {kagi }}^{(2)}$ & 39.2 & 39.5 & 39.7 & 39.8 & 39.9 & 40.2 & 40.3 & 40.5 & 40.7 & 41.0 & 41.3 & 41.7 & 42.1 \\
\hline$\omega_{\text {kagi }}$ & 1.55 & 1.55 & 1.55 & 1.55 & 1.56 & 1.57 & 1.59 & 1.60 & 1.62 & 1.62 & 1.60 & 1.59 & 1.59 \\
\hline
\end{tabular}

Таблица 2. Emini-Nasdaq100 futures

Статистический анализ показывает также, что $H$-волатильность является устойчивой характеристикой актива в зависимости от колебаний рынка. Иначе говоря, для «бурных» и «спокойных» дней зна- 
чения $H$-волатильности близки друг к другу, а это значит, что $H$-волатильность является своего рода фрактальной характеристикой актива (в то время как индикатором «бурных» и «спокойных» дней может служить $H$-инверсия, см. п. 5). В качестве примера возьмем Emini-SP500 futures и для $H=1.5$ обозначим через $D_{\text {renko }}^{1}$ дисперсию ряда $\xi^{(1)}(i) / H$, $D_{\text {renko }}^{2}$ - дисперсию ряда $\xi^{(2)}(i) / H^{2}$, где индекс $i$ указывает на дни из заданного выше диапазона, а величина $\xi^{(1)}(i)\left(\xi^{(2)}(i)\right)$ есть renko- $H$-волатильность соответствующего порядка для $i$-го дня. Аналогичным образом определим $D_{\text {kagi }}^{1}, D_{\text {kagi }}^{2}$. Тогда для данной статистической базы расчета

$$
D_{\text {renko }}^{1} \approx 0.07, \quad D_{\text {renko }}^{2} \approx 3, \quad D_{\text {kagi }}^{1} \approx 0.02, \quad D_{\text {kagi }}^{2} \approx 0.7
$$

Отметим, что в случаe renko дисперсия существенно больше, чем в случаe kagi. Это говорит о том, что kagi-методика работает лучше renko в определенном смысле. Для других $H$ из соответствующего диапазона дисперсия принимает близкие к указанным значения.

Для сравнения здесь же приведем результаты анализа для внутридневных цен обыкновенных акций РАО ЕЭС, торгуемых на бирже ММВБ, за 2001-2002 гг. (479 торговых дней). Также считаем, что время изменяется дискретно с интервалом 1 секунда, а в качестве $X(t)$ берем цену последней сделки в момент $t$. Значение $H$ измеряется в копейках, а соответствующие характеристики, усредненные по $H$ для $H \in[1.1,2.4]$ с шагом 0.1 , даны в таблице 3 .

\begin{tabular}{|c|c|c|c|c|c|c|c|c|}
\hline$\xi^{(1)} / H$ & $\xi^{(2)} / H^{2}$ & $\sigma_{\text {renko }}$ & $\sigma_{\text {renko }}^{(2)}$ & $\eta^{(1)} / H$ & $\eta^{(2)} / H^{2}$ & $\sigma_{\text {kagi }}$ & $\sigma_{\text {kagi }}^{(2)}$ & $\omega_{\text {kagi }}$ \\
\hline 1.99 & 5.95 & 0.170 & 0.169 & 2.01 & 5.10 & 0.154 & 0.156 & 1.73 \\
\hline
\end{tabular}

Таблица 3. PAO ЕЭС

\section{7. Доказательство основных результатов.}

Д оказат ел ь с т о ле м м ы 1 . Для случая $H$-флуктуации построим индуктивно следующую последовательность моментов остановки:

$$
\tilde{\rho}_{0}=0, \ldots, \quad \tilde{\rho}_{i+1}=\inf \left\{t \geqslant \tilde{\rho}_{i}:\left|X(t)-X\left(\tilde{\rho}_{i}\right)\right|=H\right\}
$$

В силу непрерывности реализаций $X$ очевидно, что для некоторого конечного $\widetilde{M}=\widetilde{M}_{T}(H, X)$ мы остановимся, т.е. $\left|X\left(\widetilde{\rho}_{\widetilde{M}}\right)-X(u)\right|<H$ для $u \in\left[\widetilde{\rho}_{\widetilde{M}}, T\right]$. Далее, из $\left(\widetilde{\rho}_{i}\right)_{i=0, \ldots, \tilde{M}}$ последовательно выделим все такие моменты $\left(\widetilde{\rho}_{i_{m}}\right)_{m=1, \ldots, M-1}$ для некоторого $M=M_{T}(H, X)$, что $i_{1}<\cdots<$ $i_{M-1}$ и выполнено

$$
\left(X\left(\widetilde{\rho}_{i_{m}}\right)-X\left(\tilde{\rho}_{i_{m}-1}\right)\right)\left(X\left(\tilde{\rho}_{i_{m}+1}\right)-X\left(\tilde{\rho}_{i_{m}}\right)\right)<0 .
$$


Обозначим $\rho_{0}^{*}=0, \rho_{0}=\widetilde{\rho}_{1}, \rho_{M}^{*}=\widetilde{\rho}_{\widetilde{M}}, \rho_{M}=T, \rho_{m}^{*}=\widetilde{\rho}_{i_{m}}, \rho_{m}=\widetilde{\rho}_{i_{m}+1}$ для $m=1, \ldots, M-1$. В итоге на интервале $[0, T]$ для некоторого случайного $M=M_{T}(H, X)$ получим последовательность $\left(\rho_{m}^{*}, \rho_{m}\right)_{m=0, \ldots, M}$. Как будет показано в дальнейшем, набор $\left(\rho_{m}^{*}\right)_{m=0, \ldots, M}$ является искомым в соотношении (5). Для случая $H$-вариации индуктивно определим следующее.

База индукции: $\tau_{0}=\inf \left\{u \in[0, T]: \max _{[0, u]} X-\min _{[0, u]} X=H\right\}$,

$$
\tau_{0}^{*}=\left\{\begin{array}{lll}
\underset{\left[0, \tau_{0}\right]}{\arg \min } X, & \text { если } & X\left(\tau_{0}\right)=\max _{\left[0, \tau_{0}\right]} X, \\
\underset{\left[0, \tau_{0}\right]}{\arg \max } X, & \text { если } & X\left(\tau_{0}\right)=\min _{\left[0, \tau_{0}\right]} X .
\end{array}\right.
$$

Шаг индукции $(n \rightarrow n+1)$ :

$$
\begin{aligned}
& \tau_{n+1}=\left\{\begin{array}{r}
\inf \left\{u \in\left[\tau_{n}, T\right]: \max _{\substack{\left[\tau_{n}, u\right] \\
\text { если }}} X\left(\tau_{n}\right)-X\left(\tau_{n}^{*}\right)=H,\right. \\
\inf \left\{u \in\left[\tau_{n}, T\right]: X(u)-\min _{\left[\tau_{n}, u\right]} X=H\right\}, \\
\text { если } X\left(\tau_{n}\right)-X\left(\tau_{n}^{*}\right)=-H,
\end{array}\right. \\
& \tau_{n+1}^{*}=\left\{\begin{array}{lll}
\underset{\left[\tau_{n}, \tau_{n+1}\right]}{\arg \max } X, & \text { если } \quad X\left(\tau_{n}\right)-X\left(\tau_{n}^{*}\right)=H, \\
\underset{\left[\tau_{n}, \tau_{n+1}\right]}{\arg \min X,} & \text { если } \quad X\left(\tau_{n}\right)-X\left(\tau_{n}^{*}\right)=-H .
\end{array}\right.
\end{aligned}
$$

Поскольку реализации $X$ непрерывны на $[0, T]$, то, очевидно, для некоторого конечного $n^{*}$ мы остановимся, т.е. $\tau_{n^{*}+1}$ не существует. Положим тогда $N=n^{*}+1, \tau_{N}=T$ и

$$
\tau_{N}^{*}=\left\{\begin{array}{lll}
\underset{\left[\tau_{n^{*}}, T\right]}{\arg \max } X, & \text { если } & X\left(\tau_{n^{*}}\right)-X\left(\tau_{n^{*}}^{*}\right)=H, \\
\underset{\left[\tau_{n^{*}}, T\right]}{\arg \min X,} & \text { если } & X\left(\tau_{n^{*}}\right)-X\left(\tau_{n^{*}}^{*}\right)=-H .
\end{array}\right.
$$

В итоге на интервале $[0, T]$ для некоторого случайного $N=N_{T}(H, X)$ получим последовательность $\left(\tau_{n}^{*}, \tau_{n}\right)_{n=0, \ldots, N}$. При этом выполнено

$$
\begin{aligned}
& \tau_{n}^{*}<\tau_{n} \leqslant \tau_{n+1}^{*}, \quad\left|X\left(\tau_{n}^{*}\right)-X\left(\tau_{n}\right)\right|=H \quad \forall n=0, \ldots, N-1, \\
& \tau_{N}^{*} \leqslant \tau_{N}=T, \quad\left|X\left(\tau_{N}^{*}\right)-X\left(\tau_{N}\right)\right|<H, \\
& \operatorname{sign}\left(X\left(\tau_{n}^{*}\right)-X\left(\tau_{n-1}^{*}\right)\right)=\operatorname{sign}\left(X\left(\tau_{n-1}\right)-X\left(\tau_{n-1}^{*}\right)\right) \\
& \quad=(-1)^{n+1} \operatorname{sign}\left(X\left(\tau_{0}\right)-X\left(\tau_{0}^{*}\right)\right) \quad \forall n=1, \ldots, N .
\end{aligned}
$$

Аналогичные соотношения выполнены и для $\left(\rho_{m}^{*}, \rho_{m}\right)_{m=0, \ldots, M}$, т.е.

$$
\begin{aligned}
& \rho_{m}^{*}<\rho_{m} \leqslant \rho_{m+1}^{*}, \quad\left|X\left(\rho_{m}^{*}\right)-X\left(\rho_{m}\right)\right|=H \quad \forall m=0, \ldots, M-1, \\
& \rho_{M}^{*} \leqslant \rho_{M}=T, \quad\left|X\left(\rho_{M}^{*}\right)-X\left(\rho_{M}\right)\right|<H, \\
& \operatorname{sign}\left(X\left(\rho_{m}^{*}\right)-X\left(\rho_{m-1}^{*}\right)\right)=\operatorname{sign}\left(X\left(\rho_{m-1}\right)-X\left(\rho_{m-1}^{*}\right)\right) \\
& \quad=(-1)^{m+1} \operatorname{sign}\left(X\left(\rho_{0}\right)-X\left(\rho_{0}^{*}\right)\right) \quad \forall m=1, \ldots, M
\end{aligned}
$$


Важно отметить, что из построения следует, что моменты $\left(\rho_{m}\right)_{m=0, \ldots, M}$, $\left(\tau_{n}\right)_{n=0, \ldots, N}$ являются марковскими, а величины $\rho_{m}^{*}, \tau_{n}^{*}$ для $m=0, \ldots, M$, $n=0, \ldots, N$ определяются соответственно в моменты $\rho_{m}, \tau_{n}$ исходя из поведения $X$ на интервалах $\left[\rho_{m-1}, \rho_{m}\right],\left[\tau_{n-1}, \tau_{n}\right]\left(\rho_{-1}=\tau_{-1}=0\right)$.

Если $H$ таково, что не существует указанных выше последовательностей, то тогда величины (1), (2) равны нулю, а значит, в качестве искомых последовательностей можно взять, например, точку 0 и доказывать нечего. В дальнейшем будем считать, что наборы $\left(\rho_{m}^{*}, \rho_{m}\right)_{m=0, \ldots, M}$, $\left(\tau_{n}^{*}, \tau_{n}\right)_{n=0, \ldots, N}$ нетривиальны, т.е. $M, N \geqslant 1$.

Рассмотрим произвольную функцию $F$, непрерывную на $[0, T]$, и определим для произвольных $\left(t_{0}, \ldots, t_{L}\right)$ таких, что $0 \leqslant t_{0} \leqslant \cdots \leqslant t_{L} \leqslant T$, функционал

$$
\Psi\left(t_{0}, \ldots, t_{L}\right)=\sum_{l=1}^{L}\left|F\left(t_{l}\right)-F\left(t_{l-1}\right)\right| .
$$

Для доказательства соотношений $(5),(6)$ достаточно показать, что для моментов $\left(\rho_{m}^{*}\right)_{m=0, \ldots, M},\left(\tau_{n}^{*}\right)_{n=0, \ldots, N}$, построенных для функции $F$ по указанному выше алгоритму, выполнено

$$
\Psi\left(\rho_{0}^{*}, \ldots, \rho_{M}^{*}\right)=U_{T}(H, F), \quad \Psi\left(\tau_{0}^{*}, \ldots, \tau_{N}^{*}\right)=V_{T}(H, F) .
$$

Докажем сначала, что $\Psi\left(\tau_{0}^{*}, \ldots, \tau_{N}^{*}\right)=V_{T}(H, F)$. Рассмотрим произвольное $\left(t_{0}, \ldots, t_{L}\right) \in \mathbf{T}_{2}$ и покажем, что $\Psi\left(t_{0}, \ldots, t_{L}\right) \leqslant \Psi\left(\tau_{0}^{*}, \ldots, \tau_{N}^{*}\right)$. Если существует $i=1, \ldots, L-1$ такое, что

$$
\left(F\left(t_{i}\right)-F\left(t_{i-1}\right)\right)\left(F\left(t_{i+1}\right)-F\left(t_{i}\right)\right)>0
$$

то точку $t_{i}$ из $\left(t_{0}, \ldots, t_{L}\right)$ выкидываем. И так далее, пока не получим некоторое разбиение $\left(s_{0}, \ldots, s_{M}\right) \in \mathbf{T}_{2}$ такое, что $\left(s_{0}, \ldots, s_{M}\right) \subseteq\left(t_{0}, \ldots, t_{L}\right)$ и выполнено

$$
\left(F\left(s_{i}\right)-F\left(s_{i-1}\right)\right)\left(F\left(s_{i+1}\right)-F\left(s_{i}\right)\right)<0 \quad \forall i=1, \ldots, M-1 .
$$

При этом, очевидно, $\Psi\left(t_{0}, \ldots, t_{L}\right)=\Psi\left(s_{0}, \ldots, s_{M}\right)$. Положим для определенности $F\left(\tau_{1}^{*}\right)-F\left(\tau_{0}^{*}\right)>0$ и рассмотрим все $\tau_{i}^{*} \in\left[0, s_{1}\right]$, пусть это точки $\left(\tau_{0}^{*}, \ldots, \tau_{I}^{*}\right)$. Заметим, что это множество не пусто, так как $\tau_{0}^{*} \in\left[0, s_{1}\right]$. Возможны два случая.

Случай 1: $F\left(s_{1}\right)-F\left(s_{0}\right)>0$. Если $I$ - нечетное число, то в разбиении $\left(s_{0}, \ldots, s_{M}\right)$ меняем точки $s_{0}, s_{1}$ на $\tau_{0}^{*}, \ldots, \tau_{I}^{*}$. Если $I$ - четное число (в частности 0 ), то в случае $F\left(s_{1}\right)-F\left(\tau_{I}^{*}\right) \geqslant H$ точку $s_{0}$ меняем на $\tau_{0}^{*}, \ldots, \tau_{I}^{*}$, иначе, т.е. $F\left(s_{1}\right)-F\left(\tau_{I}^{*}\right)<H$ (заметим, что тогда $I>1$ ), точки $s_{0}, s_{1}$ меняем на $\tau_{0}^{*}, \ldots, \tau_{I-1}^{*}$.

Случай 2: $F\left(s_{1}\right)-F\left(s_{0}\right)<0$. Если $I$ - четное число, то в разбиении $\left(s_{0}, \ldots, s_{M}\right)$ меняем точки $s_{0}, s_{1}$ на $\tau_{0}^{*}, \ldots, \tau_{I}^{*}$. Если $I-$ нечетное 
число, то в случае $F\left(\tau_{I}^{*}\right)-F\left(s_{1}\right) \geqslant H$ точку $s_{0}$ меняем на $\tau_{0}^{*}, \ldots, \tau_{I}^{*}$, иначе, т.е. в случае $F\left(\tau_{I}^{*}\right)-F\left(s_{1}\right)<H$, точки $s_{0}, s_{1}$ меняем на $\tau_{0}^{*}, \ldots, \tau_{I-1}^{*}$.

В итоге для обоих случаев получаем разбиение $\left(u_{0}, \ldots, u_{P}\right) \in \mathbf{T}_{2}$, где $\left(u_{0}, \ldots, u_{J}\right)=\left(\tau_{0}^{*}, \ldots, \tau_{J}^{*}\right),\left(u_{J+1}, \ldots, u_{P}\right)=\left(s_{J^{\prime}}, \ldots, s_{M}\right), J \in\{I-1, I\}$, $J^{\prime} \in\{1,2\}$ из построения. При этом $\Psi\left(u_{0}, \ldots, u_{P}\right) \geqslant \Psi\left(s_{0}, \ldots, s_{M}\right)$ и

$$
\left(F\left(u_{i}\right)-F\left(u_{i-1}\right)\right)\left(F\left(u_{i+1}\right)-F\left(u_{i}\right)\right)<0 \quad \forall i=1, \ldots, P-1 .
$$

Далее для интервала $\left[u_{J}, u_{J+1}\right]$ проведем следующее дробление. Для определенности положим $F\left(u_{J+1}\right)-F\left(u_{J}\right)>0$ и рассмотрим все $\tau_{i}^{*} \in\left(u_{J}, u_{J+1}\right]$. Пусть это точки $\left(\tau_{J+1}^{*}, \ldots, \tau_{J+Q}^{*}\right)$ для некоторого $Q \geqslant 1$ (если таких не найдется, то точку $u_{J+1}$ заменяем на $\tau_{J+1}^{*}$, при этом, очевидно, $\left.\tau_{J+1}^{*} \in\left(u_{J}, u_{J+2}\right), F\left(\tau_{J+1}^{*}\right) \geqslant F\left(u_{J+1}\right)\right)$. Если $Q$ - нечетное число, то точку $u_{J+1}$ заменяем на $\left(\tau_{J+1}^{*}, \ldots, \tau_{J+Q}^{*}\right)$. Если $Q$ - четное, то в случае $F\left(u_{J+1}\right)-F\left(\tau_{J+Q}^{*}\right) \geqslant H$ к исходному разбиению добавляем $\left(\tau_{J+1}^{*}, \ldots, \tau_{J+Q}^{*}\right)$, иначе, т.е. в случае $F\left(u_{J+1}\right)-F\left(\tau_{J+Q}^{*}\right)<H$, меняем $u_{J+1}$ на $\left(\tau_{J+1}^{*}, \ldots, \tau_{J+Q-1}^{*}\right)$. В итоге получим разбиение $\left(w_{0}, \ldots, w_{K}\right) \in \mathbf{T}_{2}$, где $\left(w_{0}, \ldots, w_{J+Q^{\prime}}\right)=\left(\tau_{0}^{*}, \ldots, \tau_{J+Q^{\prime}}^{*}\right),\left(w_{J+Q^{\prime}+1}, \ldots, w_{K}\right) \subseteq\left(u_{J+1}, \ldots, u_{P}\right)$, $Q^{\prime} \in\{Q-1, Q\}$, при этом $\Psi\left(w_{0}, \ldots, w_{K}\right) \geqslant \Psi\left(u_{0}, \ldots, u_{P}\right)$ и

$$
\left(F\left(w_{i}\right)-F\left(w_{i-1}\right)\right)\left(F\left(w_{i+1}\right)-F\left(w_{i}\right)\right)<0 \quad \forall i=1, \ldots, K-1 .
$$

Аналогичную процедуру проводим с интервалом $\left[w_{J+Q^{\prime}}, w_{J+Q^{\prime}+1}\right]$ (если $J+Q^{\prime}+1=K$, то берем $\left.\left[w_{J+Q^{\prime}}, T\right]\right)$ и так далее до тех пор, пока в итоге не получим искомое разбиение $\left(\tau_{0}^{*}, \tau_{1}^{*}, \ldots, \tau_{N}^{*}\right)$; т.е. выполнено

$$
\Psi\left(t_{0}, \ldots, t_{L}\right) \leqslant \Psi\left(\tau_{0}^{*}, \ldots, \tau_{N}^{*}\right) \quad \forall\left(t_{0}, \ldots, t_{L}\right) \in \mathbf{T}_{2},
$$

а значит, $V_{T}(H, F) \leqslant \Psi\left(\tau_{0}^{*}, \ldots, \tau_{N}^{*}\right)$. Обратное неравенство очевидно.

Докажем теперь, что $\Psi\left(\rho_{0}^{*}, \ldots, \rho_{M}^{*}\right)=U_{T}(H, F)$. Для этого определим функцию $\tilde{F}$ следуюшим образом:

$$
\tilde{F}(t)=\left\{\begin{array}{l}
F\left(\tilde{\rho}_{i}\right), \quad \text { если } t \in\left[\tilde{\rho}_{i}, \tilde{\rho}_{i+1}\right), \quad i=0, \ldots, \widetilde{M}-1, \\
F\left(\widetilde{\rho}_{\widetilde{M}}\right), \quad \text { если } t \in\left[\tilde{\rho}_{\widetilde{M}}, T\right] .
\end{array}\right.
$$

С одной стороны, $\left(\tilde{\rho}_{0}, \ldots, \tilde{\rho}_{\widetilde{M}}\right) \in \mathbf{T}_{1} ;$ с другой стороны, для произвольного разбиения $\left(t_{0}, t_{1}, \ldots, t_{K}\right) \in \mathbf{T}_{1}$ выполнено следующее:

$$
\forall k=0, \ldots, K \quad \exists m=m(k)=0, \ldots, \widetilde{M}: F\left(t_{k}\right)=F\left(\tilde{\rho}_{m}\right) .
$$

Отсюда следует, что $U_{T}(H, F)=U_{T}(H, \widetilde{F})$. Но для кусочно-постоянной функции $\widetilde{F}$ с конечным числом (так как $F$ непрерывна) интервалов постоянства неравенство $\Psi\left(\rho_{0}^{*}, \ldots, \rho_{M}^{*}\right) \geqslant U_{T}(H, \widetilde{F})$ выполнено очевидным образом. Действительно, набор $\left(\rho_{0}^{*}, \ldots, \rho_{M}^{*}\right)$ получается из $\left(\tilde{\rho}_{0}, \ldots, \widetilde{\rho}_{\widetilde{M}}\right)$ выкидыванием точек $\tilde{\rho}_{i}$, для которых

$$
\left(F\left(\tilde{\rho}_{i}\right)-F\left(\tilde{\rho}_{i-1}\right)\right)\left(F\left(\tilde{\rho}_{i+1}\right)-F\left(\tilde{\rho}_{i}\right)\right)>0 .
$$


Очевидно также, что $\Psi\left(\rho_{0}^{*}, \ldots, \rho_{M}^{*}\right) \leqslant U_{T}(H, \widetilde{F})$ и, следовательно, $\Psi\left(\rho_{0}^{*}, \ldots, \rho_{M}^{*}\right)=U_{T}(H, \widetilde{F})=U_{T}(H, F)$. Лемма 1 доказана.

3 а м е ч а н и е. Величины $M_{T}(H, X), N_{T}(H, X)$ тесно связаны с аппаратом кусочно-монотонных приближений. А именно: непрерывная на $[0, T]$ функция $f$ является кусочно-монотонной порядка $n \in \mathbf{N}=$ $\{1,2, \ldots\}$, если существует разбиение $\left(t_{0}, \ldots, t_{n}\right)$ такое, что $0=t_{0}<$ $t_{1}<\cdots<t_{n}=T$ и $f$ монотонна на каждом интервале $\Delta_{i}=\left[t_{i-1}, t_{i}\right]$, $i=1, \ldots, n$, и не является такой на любой паре соседних отрезков $\Delta_{i}$ (см. [4]). Множество таких функций будем обозначать $\Sigma_{n}$. Для функции $(F(t))_{t \in[0, T]}$ при данном натуральном $n$ будем рассматривать ее наилучшее приближение элементами семейства $\Sigma_{n}$, которое определяется соотношением

$$
M_{n}(F)=\inf _{f \in \Sigma_{n}} \sup _{t \in[0, T]}|F(t)-f(t)|
$$

В работе [3] было показано, что для любой непрерывной на $[0, T]$ функции $F$ выполнено

$$
N_{T}(H, F)=\min \left\{n \in \mathbf{N}: M_{n}(F)<\frac{H}{2}\right\} .
$$

Для величины $M_{T}(H, F)$ также можно установить очевидным образом, что $M_{T}(H, F)$ есть порядок кусочно-монотонной функции $\widehat{F}$, где функция $\widehat{F}$ однозначным образом определяется по значениям $\left(F\left(\tilde{\rho}_{i}\right)\right)_{i=0, \ldots, \widetilde{M}}$ $\left(\left(\tilde{\rho}_{i}\right)_{i=0, \ldots, \widetilde{M}}-\right.$ последовательность, о которой говорится в доказательстве леммы 1). А именно:

$$
\widehat{F}(t)= \begin{cases}F\left(\tilde{\rho}_{i}\right)+\left(F\left(\widetilde{\rho}_{i+1}\right)-F\left(\widetilde{\rho}_{i}\right)\right) \frac{t-\widetilde{\rho}_{i}}{\widetilde{\rho}_{i+1}-\widetilde{\rho}_{i}}, & t \in\left[\widetilde{\rho}_{i}, \tilde{\rho}_{i+1}\right], \\ F\left(\tilde{\rho}_{\widetilde{M}}\right), & i=0, \ldots, \widetilde{M}-1, \\ & t \in\left[\widetilde{\rho}_{\widetilde{M}}, T\right] .\end{cases}
$$

Указанная взаимосвязь показывает, в частности, что величины $M_{T}(H, F), \quad N_{T}(H, F)$ определены корректным образом, т.е. зависят только от функции $F$ и значений $H, T$.

Д о к а з а т е л с т в о т е о ре мы. Докажем сначала теорему для случая $\sigma=1$. Пусть $\left(\rho_{m}^{*}, \rho_{m}\right)_{m=0, \ldots, M},\left(\tau_{n}^{*}, \tau_{n}\right)_{n=0, \ldots, N}-$ моменты времени, о которых говорится в лемме 1 . Тогда из (33) следует, что для любого $m=1, \ldots, M-1$

$$
\begin{aligned}
\left|W_{\rho_{m}^{*}}-W_{\rho_{m-1}^{*}}\right|^{p} & =\left|W_{\rho_{m}^{*}}-W_{\rho_{m}}+W_{\rho_{m}}-W_{\rho_{m-1}}+W_{\rho_{m-1}}-W_{\rho_{m-1}^{*}}\right|^{p} \\
& =\left(2 H+\left(W_{\rho_{m}}-W_{\rho_{m-1}}\right) \operatorname{sign}\left(W_{\rho_{m}^{*}}-W_{\rho_{m-1}^{*}}\right)\right)^{p} .
\end{aligned}
$$


Поскольку величина $\operatorname{sign}\left(W_{\rho_{m}^{*}}-W_{\rho_{m-1}^{*}}\right)$ полностью определяется значениями $\left(W_{t}\right)_{t \in\left[\rho_{m-1}, \rho_{m}\right]}$, а $\left(\rho_{m}\right)$ - последовательность марковских моментов, то в силу свойства строгой марковости винеровского процесса величины $\left(\left|W_{\rho_{m}^{*}}-W_{\rho_{m-1}^{*}}\right|^{p}\right)_{m=1, \ldots, M-1}$ являются независимыми и одинаково распределенными. Так как $M=M_{T}(H, X) \rightarrow+\infty$ (п.н.) при $T \rightarrow+\infty$, a $\left|W_{\rho_{M}^{*}}-W_{\rho_{M-1}^{*}}\right|^{p}=\left(2 H+\left(W_{\rho_{M}}-W_{\rho_{M-1}}\right) \operatorname{sign}\left(W_{\rho_{M}^{*}}-W_{\rho_{M-1}^{*}}\right)+\varepsilon_{M}\right)^{p}$, где величина $\varepsilon_{M}$ ограничена $\left(-2 H<\varepsilon_{M}<0\right)$, то в силу усиленного закона больших чисел

$$
\frac{\sum_{m=1}^{M}\left|W_{\rho_{m}^{*}}-W_{\rho_{m-1}^{*}}\right|^{p}}{M} \longrightarrow \mathbf{E}\left|W_{\rho_{1}^{*}}-W_{\rho_{0}^{*}}\right|^{p} \quad \text { (п.н.) } \quad \text { при } T \rightarrow+\infty .
$$

Аналогично доказывается, что

$$
\frac{\sum_{n=1}^{N}\left|W_{\tau_{n}^{*}}-W_{\tau_{n-1}^{*}}\right|^{p}}{N} \longrightarrow \mathbf{E}\left|W_{\tau_{1}^{*}}-W_{\tau_{0}^{*}}\right|^{p} \quad \text { (п.н.) } \quad \text { при } T \rightarrow+\infty .
$$

Найдем сначала $\mathbf{E}\left|W_{\rho_{1}^{*}}-W_{\rho_{0}^{*}}\right|^{p}$. Для этого рассмотрим последовательность $\left(\alpha_{k}\right)_{k=1,2, \ldots}$ независимых одинаково распределенных величин с распределением

$$
\alpha_{k}=\left\{\begin{array}{rr}
1, & 0.5 \\
-1, & 0.5
\end{array}\right.
$$

Обозначим также $\beta_{n}=\sum_{k=1}^{n} \alpha_{k}, n=1,2, \ldots$, и рассмотрим случайную величину

$$
\nu=\min \left\{n \geqslant 1: \beta_{n}=n-2\right\} .
$$

Заметим, что $\nu$ - момент первого падения $\beta_{n}$. Поскольку, как легко понять из (34), $\left|W_{\rho_{1}^{*}}-W_{\rho_{0}^{*}}\right|^{p} \stackrel{\text { Law }}{=}\left(2 H+\beta_{\nu} H\right)^{p}$, то из того, что $\beta_{\nu}=\nu-2$, а $\mathbf{E} \nu^{p}=\sum_{n=1}^{\infty} n^{p} / 2^{n}$, следует (13) для $\sigma=1$.

Для доказательства (14) обозначим

$$
\theta=\inf \left\{u \geqslant 0: \max _{[0, u]} W-W_{u}=H\right\} .
$$

В силу марковости моментов $\tau_{0}, \tau_{1}$ легко понять, что

$$
\left(W_{\tau_{1}}-W_{\tau_{0}}\right) \operatorname{sign}\left(W_{\tau_{1}^{*}}-W_{\tau_{0}^{*}}\right) \stackrel{\text { Law }}{=} W_{\theta},
$$

a значит, $\mathbf{E}\left|W_{\tau_{1}^{*}}-W_{\tau_{0}^{*}}\right|^{p}=\mathbf{E}\left(2 H+W_{\theta}\right)^{p}$, что доказывает искомое.

Для доказательства теоремы для случая произвольного $\sigma>0$ достаточно воспользоваться свойством автомодельности винеровского процесса: $\left(\sigma W_{t} ; 0 \leqslant t \leqslant T\right) \stackrel{\text { Law }}{=}\left(W_{t} ; 0 \leqslant t \leqslant \sigma^{2} T\right)$. Отсюда следует, что

$$
\begin{aligned}
& \left(M_{T}(H, \sigma W), U_{T}^{(p)}(H, \sigma W)\right) \stackrel{\text { Law }}{=}\left(M_{\sigma^{2} T}(H, W), U_{\sigma^{2} T}^{(p)}(H, W)\right), \\
& \left(N_{T}(H, \sigma W), V_{T}^{(p)}(H, \sigma W)\right) \stackrel{\text { Law }}{=}\left(N_{\sigma^{2} T}(H, W), V_{\sigma^{2} T}^{(p)}(H, W)\right) .
\end{aligned}
$$

Теорема доказана. 
3 а м е ч а и е. Для случаев $p=1,2$ найдем значения $R_{W}(p)$, $K_{W}(p)$. Легко подсчитать, что $R_{W}(1)=2, R_{W}(2)=6$. Для того чтобы найти $K_{W}(1), K_{W}(2)$, рассмотрим величину

$$
\theta^{\prime}=\inf \left\{u \geqslant 0:\left|W_{u}\right|=H\right\}
$$

Поскольку в силу теоремы Леви (см. $\left[6\right.$, с. 211]) $\left|W_{t}\right| \stackrel{\text { Law }}{=} \max _{[0, t]} W-W_{t}$, то величины $\theta^{\prime}$ и $\theta$ имеют одинаковые распределения, а значит, $\mathbf{E} \theta=\mathbf{E} \theta^{\prime}$. Но в силу тождества Вальда $\mathbf{E} \theta^{\prime}=\mathbf{E} W_{\theta^{\prime}}^{2}=H^{2}$, поэтому $\mathbf{E} W_{\theta}^{2}=H^{2}$, $\mathbf{E} W_{\theta}=0$, откуда следует, что $K_{W}(1)=2, K_{W}(2)=5$.

Д оказатель с т о ле м мы 2. Рассмотрим сначала случай $\sigma=1$. Пусть $\left(\tilde{\rho}_{m}\right)_{m=0, \ldots, \tilde{M}},\left(\tau_{n}\right)_{n=0, \ldots, N}$ - марковские моменты, о которых говорится в лемме 1 . Тогда каждая из последовательностей $\left(\tilde{\rho}_{m}-\tilde{\rho}_{m-1}\right)_{m=1, \ldots, \tilde{M}}$ и $\left(\tau_{n}-\tau_{n-1}\right)_{n=1, \ldots, N}$ представляет собой последовательность независимых одинаково распределенных случайных величин, при этом

$$
\left(\tilde{\rho}_{m}-\tilde{\rho}_{m-1}\right) \stackrel{\text { Law }}{=} \theta^{\prime}, \quad\left(\tau_{n}-\tau_{n-1}\right) \stackrel{\text { Law }}{=} \theta
$$

где $\theta, \theta^{\prime}$ определяются из соотношений $(35),(38)$ соответственно. Поскольку $\mathbf{E} \theta^{\prime}=\mathbf{E} \theta=H^{2}$ (см. выше), а при $T \rightarrow+\infty$ выполнено $\widetilde{M}=\widetilde{M}_{T}(H, W) \rightarrow+\infty, N=N_{T}(H, W) \rightarrow+\infty$ (п.н.), то в силу усиленного закона больших чисел немедленно следует соотношение (16) для $\sigma=1$ и

$$
\lim _{T \rightarrow+\infty} \frac{T}{\widetilde{M}_{T}(H, W)}=H^{2} \quad \text { (п.н.). }
$$

Для доказательства (15) в случае $\sigma=1$ рассмотрим $U_{T}^{(p)}(H, W)$ для $p=1$. Несложно понять, что

$$
U_{T}^{(1)}(H, W)=\widetilde{M}_{T}(H, W) H .
$$

Последнее равенство в совокупности с (13) и (39) дает искомое. Доказательство леммы для произвольного $\sigma>0$ следует из (36), (37).

Доказательст во у тверж ден ия 1. Докажем утверждение сначала для случая renko. Из (21), (33) следует

$$
\begin{aligned}
\tilde{Y}_{T}^{R}(H, X) & =\sum_{m=1}^{M_{T}(H, X)}(-1)^{m+1} \operatorname{sign}\left(X\left(\rho_{0}\right)-X\left(\rho_{0}^{*}\right)\right) \int_{0}^{T} \chi_{\left[\rho_{m-1}, \rho_{m}\right)}(t) d X(t) \\
& =\sum_{m=1}^{M_{T}(H, X)}(-1)^{m+1} \operatorname{sign}\left(X\left(\rho_{0}\right)-X\left(\rho_{0}^{*}\right)\right)\left(X\left(\rho_{m}\right)-X\left(\rho_{m-1}\right)\right. \\
& \left.+X\left(\rho_{m}^{*}\right)-X\left(\rho_{m}^{*}\right)+X\left(\rho_{m-1}^{*}\right)-X\left(\rho_{m-1}^{*}\right)\right) \\
& =\sum_{m=1}^{M_{T}(H, X)}\left(\left|X\left(\rho_{m}^{*}\right)-X\left(\rho_{m-1}^{*}\right)\right|-2 H\right)+\varepsilon_{T}^{R}(H, X),
\end{aligned}
$$


где $\varepsilon_{T}^{R}(H, X)=H+\tilde{\varepsilon}_{T}^{R}(H, X)$, a $\left|\tilde{\varepsilon}_{T}^{R}(H, X)\right|=\left|X\left(\rho_{M_{T}(H, X)}^{*}\right)-X\left(\rho_{M_{T}(H, X)}\right)\right|$. Тогда в силу (33) выполнено $0<\varepsilon_{T}^{R}(H, X)<2 H$. Случай kagi доказывается аналогично с той лишь разницей, что $0<\varepsilon_{T}^{K}(H, X) \leqslant H$. Утверждение 1 доказано.

Доказательст во утверждения 2. Если $R(H) \geqslant 2$, то из (40) следует

$$
Y_{T}^{R}(H, X)=\left(\xi_{T}(H, X)-2 H\right) M_{T}(H, X)+\varepsilon_{T}^{R}(H, X),
$$

откуда очевидным образом получаем

$$
\begin{aligned}
\lim _{T \rightarrow+\infty} \mathbf{E} y_{T}^{R}(H, X) & =\lim _{T \rightarrow+\infty} \mathbf{E} \xi_{T}(H, X)-2 H+\lim _{T \rightarrow+\infty} \mathbf{E} \frac{\varepsilon_{T}^{R}(H, X)}{M_{T}(H, X)} \\
& =(R(H)-2) H .
\end{aligned}
$$

Аналогично, если $R(H)<2$, то из (40) следует

$$
Y_{T}^{R}(H, X)=\left(2 H-\xi_{T}(H, X)\right) M_{T}(H, X)-\varepsilon_{T}^{R}(H, X),
$$

а значит, $\lim _{T \rightarrow+\infty} \mathbf{E} y_{T}^{R}(H, X)=(2-R(H)) H$.

Утверждение 2 доказано. Утверждение 3 доказывается аналогично.

Автор выражает благодарность А.Н. Ширяеву и Б. С. Кашину за полезные замечания при обсуждении работы.

\section{СПИСОК ЛИТЕРАТУРЫ}

1. Дуади Р., Йор М., Ширяев А. Н. О вероятностных характеристиках величин «падения» в стандартном броуновском движении. - Теория вероятн. и ее примен., 1999 , т. 44 , в. 1 , c. $3-13$.

2. Кашин Б. С., Пастухов С. В. О краткосрочном прогнозировании на рынке ценных бумаг. - Докл. РАН, 2002, т. 387, № 6, с. 754-756.

3. Пастухов С. В. Об $H$-волатильности в финансовой математике. - Успехи матем. наук, 2003, т. 58, № 1, с. 191-192.

4. Севастьянов E. A. Кусочно монотонная аппроксимация и $\Phi$-вариации. - Anal. Math., 1975, v. 1, p. 141-164.

5. Ширяев A. Н. Основы стохастической финансовой математики. Т. 1. М.: Фазис, $1998,489 \mathrm{c.}$

6. Karatzas I., Shreve S.E. Brownian Motion and Stochastic Calculus. New York: Springer-Verlag, 1998, $470 \mathrm{p}$.

7. Nison S. Beyond Candlesticks: New Japanese Charting Techniques Revealed. New York: Wiley, 1994, 280 p. 